Acta Cryst. (1994). B50, 1-3

\title{
Editorial
}

\section{An historical perspective}

It is an honour to be writing an Editorial that will preface the Golden Jubilee volume of Section B of Acta Crystallographica. Indeed, it is appropriate to pay immediate tribute to those far sighted scientists who first promulgated the International Union of Crystallography and then established Acta as their own first act on behalf of the expanding international family of crystallographers. It is worth repeating the original vision as expressed by Paul Ewald in the Editorial Preface of Acta Cryst. (1948), 1, 1-2: 'Acta is intended to offer a central place for publication and discussion of all research in this vast and ever-expanding field. It borders, naturally, on pure physics, chemistry, biology, mineralogy, technology and also on mathematics, but is distinguished by being concerned with the methods and results of investigating the arrangement of atoms in matter, particularly when that arrangement has regular features'. The original vision had its practical side as well: Volume 1 of Acta was offered at an annual subscription of $£ 2$. 10 s. $0 \mathrm{~d}$. (£2.50 in modern parlance) or $\$ 10$. This low rate was made possible by financial contributions from companies and organizations on fixed-term agreements: sponsorship is not just a modern phenomenon and we owe gratitude to those sponsors in their turn.

The success of Acta led, in 1968, to a division into Section $A$ (theoretical and general crystallography, crystal physics and diffraction), with structural crystallography and crystal chemistry being covered by the new Section B. In this same year, the Journal of Applied Crystallography also commenced publication. The rapidly increasing volume of papers describing crystal structure determinations led to the creation in 1983 of Section $C$, to carry much of the structural output of Section B together with that of Crystal Structure Communications, originated by the Crystallography Group of the University of Parma. Section $B$ was renamed Structural Science with a brief to publish papers 'in which the relationship of atomic arrangement to chemical, physical, biological, metallurgical, mineralogical and other important properties is considered' [Editorial, Acta Cryst. (1982), B38, 1-2]. This brief was further refined [Acta Cryst. (1983), B39, 139] and over the past decade Section $B$ has developed its own independent scientific flavour, publishing novel structural science across the complete chemical spectrum from metals and alloys to proteins and viruses. In 1990 it was proposed that Acta should recognize the fundamental contributions of crystallography in the biological sciences and a new Section $D$, covering biological, particularly macromolecular, structural science, commenced publication in 1993. This logical and, perhaps, overdue development has, however, removed most structural biology from the remit of Section B.

This brief history of Section $B$ confirms Ewald's view in that first Editorial [Acta Cryst. (1948), 1, 1-2] that: 'The best scheme for the publication of scientific investigations is a problem of outstanding importance for the sound development of science, and is particularly acute in those fields which touch on many different branches of study' (present authors italics). It is precisely because crystal structure analysis has underpinned so many advances in chemistry, biology and materials science that diversification of the original Section $B$ has occurred. Indeed, it is for this reason, among others, that the chemical, biological and materials science literature contains many papers that might otherwise have appeared in Section B. This is not a stricture: such papers maintain and enhance the perception of crystallography in those sciences that it touches. However, it does mean that Section $B$ operates in an increasingly competitive environment and, to improve and develop, it must continue to encourage its own special approach to structural science, its own special themes and, particularly, provide forms of presentation that are best suited to those themes.

\section{The scientific scope of Section $B$}

It is appropriate for this Editorial to identify those areas of. research that now fall within the remit of Section $B$. As a consequence, we may hope to define more clearly the objectives for Volume B50 and beyond.

Firstly, it is clear that Section B will publish papers in which structure, the three-dimensional arrangement of atoms in matter, is the primary focus of the work reported. Secondly, Section $B$ must continue to have a broad chemical coverage that now includes metals and alloys, inorganics and minerals, organometallics and metal complexes and organic systems.

Any further definition of the scientific scope of Section $B$ is best obtained from a survey of the broad fields of study that are typically reported there, or ought to be reported there. These include, inter alia: structural systematics, correlations and comparisons in covalently bonded, hydrogen-bonded, non-bonded and ionic systems; correlations of structure with physicochemical and other properties as obtained by experimental, theoretical or computational procedures; the analysis of electron-density distributions; the interpretation of atomic displacement parameters in a structural context; the results of novel methods of structure determination; the application of structural information to the design of novel materials, novel active molecules and in the study of molecular recognition phenomena; aspects of 
crystal engineering, polymorphism, solid-state reactivity and related topics; novel methodologies for the storage, representation, analysis and visualization of structural information. Original structure determinations may be the basis of, or form part of, any report in these fields. Reports of individual structure determination(s) are also acceptable as the sole subject matter of a Section $B$ article, provided that the scientific scope of the structural description and interpretation is significantly enhanced over the norms established for Section $C$ of Acta.

This is a long and diverse list of topics applied across a broad chemical spectrum but, within it, some themes emerge. Those themes, the themes of Section $B$, are the acquisition of significant structural knowledge from novel experimental observations or by analysis of existing data, the correlation of structural knowledge with physico-chemical or other properties and the application of this knowledge to solve problems in the structural domain.

\section{Categories of contributions to Section $B$}

To sustain a lively environment for its authors and readers, every scientific journal must provide a variety of publication formats and time schedules. Acta Crystallographica has always incorporated new ideas in this important area and, from Volume B50, Section B will promote structural science in four main styles of presentation.

The classical format of scientific writing is the original full-length paper. Articles in this style will remain the core of Section $B$ and will be identified within the journal as Research Papers. As now, full papers should be submitted to a member of the International Coeditorial Board who will process the paper through refereeing, decide on acceptance or rejection and handle any revisions that may be necessary.

Often, however, a short format is desirable for the presentation of topics of more limited scope, or for preliminary announcements of novel research findings. Here, the brevity of the report is often coupled with a need for rapid publication. Recently, Section $B$ has offered both Short Communications and Fast Communications as possible routes for shorter articles. From Volume B50, these papers will simply be identified within the journal as Short Communications. However, and importantly, all Communications will be processed and printed as rapidly as possible, within the obvious time constraints of a bimonthly journal. In order to achieve this aim, desktop publishing methods or prioritized typesetting will be employed by the Editorial Office. This means that a length limitation of $c a$ two journal pages (1500 words or 6-7 double-spaced manuscript pages) will be rigidly applied, together with an upper limit of two figures and two tables of appropriate size. A lively and vigorous Communications section is seen as an important feature of Section $B$. However, it is unlikely that reports of individual structure determinations will be acceptable here, since Section $C$ exists for this purpose. All Communications will be handled by the Editor, at least for an initial period, and will be refereed rapidly but thoroughly.

Finally, we address the topic of review material in Section B. In recent years, Acta has incorporated specially commissioned Lead Articles: authoritative, comprehensive and forward looking reviews of major areas of current research. These articles have been well researched, well presented and well received and this tradition will continue in Section B. Indeed, some excellent Lead Articles are already scheduled for imminent publication. Possible topics for comprehensive coverage as Lead Articles, together with suggested name(s) of possible author(s), are always welcomed by the Editor who, as part of established practice, will discuss these suggestions with the Editorial Board. Lead Articles will continue to be highlighted on the cover of the relevant issue of Section $B$, will be clearly identified within the journal and, from Volume B50, will carry brief biographical details of their author(s).

The preparation of a Lead Article is, however, a major undertaking and such articles may be occasional in Section B. Nevertheless, structural science is a broad and rich field of endeavour that crosses many subject boundaries in every issue of the journal. This breadth of activity creates a need for experts in specific sub-areas to keep the readership alerted to recent important research developments and their implications for the future of their speciality. It is for these reasons that Section $B$, in common with Sections $A$ and $D$, is introducing a new Topical Review format.

A Topical Review in Section $B$ should be a concise and highly focused survey of current research in a relatively narrow field of structural science. It must be written so as to benefit both subject experts and also a more general audience of research scientists with an interest in structural topics. A Topical Review should not aim to be comprehensive, but a brief introduction should provide historical perspective and a brief conclusion should indicate likely future directions in the field. It is hoped that a less formal style of scientific writing can prevail in this form of publication. Topical Reviews will normally be limited to some 40 manuscript pages (about ten journal pages) and will be commissioned by the Editor, either personally or following a formal proposal by prospective author $(s)$. A letter of intent should be submitted (faxed or e-mailed) indicating the proposed topic, its perceived importance and a brief indication of the material to be covered. The letter may be sent to one or more members of the Editorial Board for comment before the full article is commissioned. Once commissioned, authors will have priority in their topic within an agreed deadline for delivery of the completed 
text. Manuscripts will be refereed in the normal way. It is hoped that publication times for Topical Reviews will be as short as possible and that direct contribution by prospective authors will become the norm for this format.

Despite the space allocated to reviews in this Editorial, necessary to the introduction of new concepts, there is no intention that this material will dominate the published journal. The primary purpose of Section $B$ is to publish original research papers and communications. However, if Section $B$ is to act as a central focus for structural science, then the inclusion of a controlled amount of review materiai is seen as essential to effective information exchange and as a catalyst for new developments.

\section{Presentation of Section $B$}

The complete family of IUCr journals has undergone a change in appearance for 1994, using modifications of ideas incorporated in the initial volume of Section $D$ (1993). These changes take advantage of modern printing technology, particularly in the use of colour, provide a clearer layout of essential journal information and incorporate a new display-style table of contents. The Editorial Staff in Chester are thanked for their advice, enthusiasm and judgement in carrying out these layout changes with a very short lead time.

\section{Production of Section $B$}

The IUCr is now seen as one of the leaders in the area of machine-readable submissions of data and manuscripts, primarily through its activities with Section $C$. Extensions of these facilities to Section $B$ are under active discussion and announcements will be made accordingly. At present the submission of formal printed manuscripts remains the norm for Section B, but authors who have used certain word-processing packages are invited to enclose diskettes with their submission. This is particularly important for Short Communications, as described in the Notes for Authors which follow this Editorial. The Notes contain the technical possibilities that are currently supported.

\section{Future directions for Section B}

This Editorial has attempted to clarify the scientific scope of Section $B$ and its role within the general framework of Acta Crystallographica. It has also set in place the philosophy and guidelines relating to the categories of contributions that will be published here. This printed issue itself illustrates the changes that have already been effected in journal presentation. The aim of the complete package is to continue the development of Section $B$ as a vigorous, varied and attractive publication medium for the very best original papers, communications and reviews in structural science. It is a personal belief that we are only just beginning to explore the subtleties of structure, their relationships to other phenomena and the ways in which structural knowledge can be applied. Since there are commonalities of approach to these activities that transcend arbitrary chemical or methodological boundaries, then the very diversity that characterizes Section $B$ can be seen as its strength. However, a journal, like the subject it represents, is a living entity that must change as the subject changes, so as to meet the needs of both authors and readers alike. Indeed, the changes discussed in this Editorial result from the comments and suggestions of many scientists in the recent past. Obviously no individual scientist can have a truly expert knowledge of all of the topics published by Section B. However, by acting on the advice of Editorial colleagues and other experts, an Editor can shape a journal that continues to respond to change in an effective manner. These at least are the hopes - let us see if we can put them into practice.

\author{
F. H. ALLEN
} Editor, Section B 